\title{
Space-Time Adaptive Processing in Bistatic Passive Radar Exploiting Complex Bayesian Learning
}

\author{
Yimin D. Zhang ${ }^{\dagger}$ and Braham Himed ${ }^{\ddagger}$ \\ $\dagger$ Center for Advanced Communications, Villanova University, Villanova, PA 19085, USA \\ $\ddagger$ Air Force Research Laboratory, AFRL/RYMD, Dayton, OH 45433, USA
}

\begin{abstract}
In this paper, we develop a new space-time adaptive processing (STAP) technique for bistatic passive radar by exploiting clutter sparsity so as to enable effective clutter suppression with a small set of data samples. The Bayesian compressive sensing (BCS) technique is utilized for sparse clutter reconstruction, and the persymmetry property of the STAP processor is used to cast the complex sparse signal recovery problem into a group sparsity formulation. This approach provides improved recovery of the clutter and, thereby, yields better STAP performance.
\end{abstract}

\section{INTRODUCTION}

Passive radar is an attractive sensing platform in many surveillance applications because of its low-cost and covert operation capability [1]. One of the major objectives in such sensing operations is to perform moving target indication (MTI). To identify moving targets, particularly those with slow velocities, from strong ground clutter, space-time adaptive processing (STAP) is considered an effective means to suppress clutter in the joint space-time domain [2].

A fundamental requirement of performing STAP is an estimated clutter covariance matrix (CCM). Conventionally, the CCM is obtained from neighboring range gates which are assumed to provide independent and identically distributed (i.i.d.) secondary, or training, samples [3], [4].

The requirement of the large number of i.i.d. secondary samples, however, may be highly impractical. Many clutter environments are only locally stationary and it is difficult to expect stationarity over a large number of range bins. This problem is particularly significant in passive radars which usually use narrowband signals and, therefore, have a poor range resolution [5]-[7]. It is thus important to improve the convergence rate by using a smaller number of secondary samples. The commonly used approaches to improve the robustness of the STAP with fewer secondary samples are to apply diagonal loading (DL) to the sample covariance matrix (SMI), or to reduce the rank of the CCM by performing principal component analysis [8], [9]. However, the convergence rate can hardly be improved beyond the clutter rank.

A more recent approach is to exploit the latest advances of sparsity recovery and compressive sensing (CS) techniques by taking advantages of the sparsity of the clutter in the angleDoppler domain [10]-[12]. It is well known that the clutter is

The work of Y. D. Zhang was supported in part by the Air Force Summer Faculty Fellowship Program under contract FA9550-09-C-0114, by a subcontract with Defense Engineering Corporation for research sponsored by the Air Force Research Laboratory under Contract FA8650-12-D-1376, and by a subcontract with Dynetics, Inc. for research sponsored by the Air Force Research Laboratory under Contract FA8650-08-D-1303. localized in this joint domain with a certain clutter ridge that is determined by the radar operation mode and antenna geometry [2], [13]. As such, sparsity-based STAP may achieve effective clutter suppression with very few secondary samples. Indeed, under certain mild assumptions, STAP can be performed with only a single sample, i.e., no secondary samples are required.

In this paper, we consider STAP in a bistatic passive radar by exploiting the sparse recovery techniques. A major issue encountered in the application of sparse recovery is that reliable recovery is guaranteed only when the so-called restricted isometry property (RIP) is satisfied [14]. Unfortunately, such a condition cannot be easily verified. Because any discrepancy between the actual and estimated clutter profile degrades the STAP performance, a more reliable CS solution is needed in the underlying problem. Toward this objective, we use the Bayesian compressive sensing (BCS) [15], [16] which reformulates the original deterministic problem as a probabilistic one to be efficiently solved with the relevance vector machine (RVM) concept [17]. Further, to effectively deal with complex scattering coefficients, the problem is formulated to exploit the group sparsity of the real and imaginary components of the same range-Doppler grid. This yields a significant performance improvement as compared to the results when the real and imaginary components are dealt separately.

Notations: We use lower-case (upper-case) bold characters to represent vectors (matrices). In particular, $\mathbf{I}_{N}$ and $\mathbf{J}_{N}$ are the $N \times N$ identity matrix and anti-diagonal matrix, respectively. (.) $)^{*},(.)^{T}$ and $(.)^{H}$ denote complex conjugate, transpose, and conjugate transpose, respectively. $\operatorname{vec}(\cdot)$ is the vectorization operator, and $\operatorname{diag}(\mathbf{x})$ denotes a diagonal matrix with the elements of $\mathbf{x}$ constituting the diagonal entries. $\|\cdot\|$ denotes the Euclidean norm of a vector, and $\otimes$ denotes the Kronecker product. $\mathrm{E}(\cdot)$ is the statistical expectation operator, and $\mathcal{N}(a, b)$ implies a Gaussian probability density function of mean $a$ and variance $b$.

\section{SYSTEM MODEL}

\section{A. Radar Geometry and Signal Model}

Consider a bistatic radar scene as depicted in Fig. 1. The transmitter is assumed to be stationary and is located at a known position $\mathbf{p}_{\mathrm{T}}=\left[p_{\mathrm{T}, x}, p_{\mathrm{T}, y}, p_{\mathrm{T}, z}\right]^{T}$. The radar receiver, which utilizes an $N$-element uniform linear array (ULA) with inter-element spacing $d$, moves in a straight line with a constant velocity.

For the convenience of notation and without loss of generality, we assume that the receiver moves in the $x$-direction 
at speed $v_{\mathrm{R}}$. The initial position of the reference sensor is assumed to be $\mathbf{p}_{\mathrm{R}}(0)=\left[p_{\mathrm{R}, x}(0), 0, H_{\mathrm{R}}\right]^{T}$.

The signal vector received at the array consists of the following terms:

$$
\mathbf{x}(t)=\mathbf{x}_{d}(t)+\mathbf{x}_{s}(t)+\mathbf{x}_{c}(t)+\mathbf{n}(t),
$$

where $\mathbf{x}_{d}(t)$ is the direct-path signal from the illuminator, $\mathbf{x}_{s}(t)$ is the desired signal, $\mathbf{x}_{c}(t)$ is the clutter, and $\mathbf{n}(t)$ is the additive noise, which is characterized as i.i.d. complex Gaussian.

Based on the known positions of the transmitter and receiver, we can perform beamforming to perfectly recover the transmitted signal waveform $s(t)$ from $\mathbf{x}(t)$ after channel decoding [7]. We assume that the direct-path component $\mathbf{x}_{d}(t)$ is subsequently removed from $\mathbf{x}(t)$.

\section{B. Desired Signal}

The desired signal is denoted as

$$
\begin{array}{r}
\mathbf{x}_{s}(t)=\frac{\sqrt{P_{\mathrm{T}}} G \sigma_{P}}{r_{\mathrm{TP}} r_{\mathrm{PR}}} s\left(t-\tau_{\mathrm{TP}}(t)-\tau_{\mathrm{PR}}(t)\right) \\
\cdot e^{-j 2 \pi f_{c}\left(\tau_{\mathrm{TP}}(t)+\tau_{\mathrm{PR}}(t)\right)} \mathbf{a}\left(\phi_{\mathrm{P}}\right),
\end{array}
$$

where $P_{\mathrm{T}}$ is the transmit power, $G$ represents a constant term, and $\sigma_{P}$ is the complex target reflection coefficient associated with the radar cross section (RCS). In addition, $f_{c}$ is the carrier frequency, $\tau_{\mathrm{TP}}(t)=r_{\mathrm{TP}}(t) / c$ and $\tau_{\mathrm{PR}}(t)=r_{\mathrm{PR}}(t) / c$ are the delays respectively corresponding to the range between the transmitter and the target, $r_{\mathrm{TP}}(t)=\left\|\mathbf{P}_{\mathrm{T}}-\mathbf{P}(t)\right\|$, and the range between the target and the receiver, $r_{\mathrm{PR}}(t)=\| \mathbf{P}_{\mathrm{R}}(t)-$ $\mathbf{P}(t) \|$, where $c$ is the velocity of light. Furthermore, ${ }^{1}$

$$
\mathbf{a}\left(\phi_{\mathrm{P}}\right)=\left[e^{j \kappa(N-1) d / 2 \sin \left(\phi_{\mathrm{P}}\right)}, \ldots, e^{-j \kappa(N-1) d / 2 \sin \left(\phi_{\mathrm{P}}\right)}\right]^{T}
$$

is the steering vector of the receive array toward the direction of the target with a cone direction-of-arrival (DOA) angle of $\phi_{\mathrm{P}}$, and $\kappa=2 \pi / \lambda$ is the wavenumber with $\lambda=c / f_{c}$ denoting the wavelength. The DOA variation during the coherent processing interval (CPI) is negligible and is thus not considered.

By performing matched filtering using the reference signal at the $n$th range bin, we obtain the matched filter output at azimuth time $t_{l}=l T, l=0, \ldots, L-1$, as

$$
\mathbf{y}_{s}^{(n)}\left(t_{l}\right)=\frac{\sqrt{P_{\mathrm{T}}} G \sigma_{P}}{r_{\mathrm{TP}} r_{\mathrm{PR}}} \rho_{s} \mathbf{a}\left(\phi_{\mathrm{P}}\right) e^{j 2 \pi \nu_{s}\left(t_{l}-(L-1) T / 2\right)},
$$

where $\rho_{s}$ is the matched filter output of $s(t)$, and $\nu_{s}=-\frac{1}{\lambda}$. $\frac{d}{d t}\left[r_{\mathrm{TP}}(t)+r_{\mathrm{PR}}(t)\right]$ is the bistatic Doppler frequency. Note that we align the phase reference center at the center of the CPI to obtain the persymmetry property.

Stack $\mathbf{y}_{s}^{(n)}\left(t_{l}\right)$ over the $L$ collected azimuthal samples as

$\tilde{\mathbf{y}}_{s}^{(n)}=\left[\left[\mathbf{y}_{s}^{(n)}\left(t_{0}\right)\right]^{T}, \ldots,\left[\mathbf{y}_{s}^{(n)}\left(t_{L-1}\right)\right]^{T}\right]^{T}=\frac{\sqrt{P_{\mathrm{T}}} G \sigma_{P}}{r_{\mathrm{TP}} r_{\mathrm{PR}}} \rho_{s} \mathbf{h}\left(\nu_{s}, \phi_{\mathrm{P}}\right)$,

where

$$
\mathbf{h}\left(\nu_{s}, \phi_{\mathrm{P}}\right)=\mathbf{b}\left(\nu_{s}\right) \otimes \mathbf{a}\left(\phi_{\mathrm{P}}\right)
$$

is the spatio-temporal signature of the target, and $\mathbf{b}\left(\nu_{s}\right)=$ $\left[e^{-j 2 \pi \nu_{s}(L-1) T / 2}, e^{-j 2 \pi \nu_{s}(L-3) T / 2}, \ldots, e^{j 2 \pi \nu_{s}(L-1) T / 2}\right]^{T}$ is the temporal signature vector of the target.

\footnotetext{
${ }^{1}$ We define the steering vector and the temporal signature vector in a symmetric form for convenience of the persymmetry formulation.
}

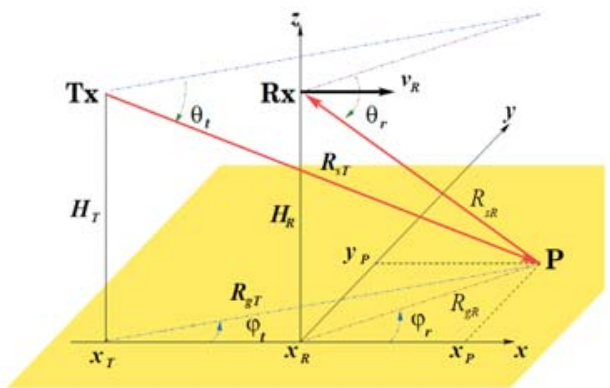

Fig. 1. Bistatic radar geometry.

\section{Clutter}

The clutter is modeled as a sum of $N_{c}$ statistically independent scatterers in range, expressed as

$$
\begin{gathered}
\mathbf{x}_{c}(t)=\sum_{i=1}^{N_{c}} \frac{\sqrt{P_{\mathrm{T}}} G \sigma_{c, i}}{r_{\mathrm{TC}, i} r_{\mathrm{CR}, i}(t)} s\left[t-\tau_{\mathrm{TC}, i}-\tau_{\mathrm{CR}, i}(t)\right] \\
\cdot e^{-j 2 \pi f_{c}\left[\tau_{\mathrm{TC}, i}+\tau_{\mathrm{CR}, i}(t)\right]} \mathbf{a}\left(\phi_{i}\right),
\end{gathered}
$$

where, similarly, $r_{\mathrm{TC}, i}$ is the range between the transmitter and the $i$ th scatterer, $r_{\mathrm{CR}, i}(t)$ is the range between the same scatterer to the receiver, $\tau_{\mathrm{TC}, i}$ and $\tau_{\mathrm{CR}, i}(t)$ are respectively the delays corresponding to $r_{\mathrm{TC}, i}$ and $r_{\mathrm{CR}, i}(t)$. In addition, $\sigma_{c, i}$ is the reflection coefficient of the $i$ th scatterer, and $\mathbf{a}\left(\phi_{i}\right)$ is its steering vector observed at the receive array.

Performing matched filtering and stacking the output over the $L$ collected azimuthal samples in the $n$th range bin yields

$$
\tilde{\mathbf{y}}_{c}^{(n)}=\sum_{i=1}^{N_{c}} \frac{\sqrt{P_{\mathrm{T}}} G \sigma_{c, i}}{r_{\mathrm{TC}, i} r_{\mathrm{CR}, i}} \rho_{s}\left(\tau_{i}^{(n)}\right) \mathbf{h}\left(\nu_{i}, \phi_{i}\right),
$$

where $\rho_{s}\left(\tau_{i}^{(n)}\right)$ is the waveform correlation coefficient associated with delay $\tau_{i}^{(n)}, \nu_{i}=-\frac{1}{\lambda} \cdot \frac{d}{d t}\left[r_{\mathrm{CR}, i}(t)-r_{\mathrm{TR}}(t)\right]$ is the Doppler frequency of the $i$ th scatterer and is determined by the scatter-receiver path and the direct-path ranges. In addition,

$$
\mathbf{h}\left(\nu_{i}, \phi_{i}\right)=\mathbf{b}\left(\nu_{i}\right) \otimes \mathbf{a}\left(\phi_{i}\right)
$$

is the spatio-temporal signature of the $i$ th scatterer, and $\mathbf{b}\left(\nu_{i}\right)=\left[e^{-j 2 \pi \nu_{i}(L-1) T / 2}, e^{-j 2 \pi \nu_{i}(L-3) T / 2}, \quad \ldots\right.$, $\left.e^{j 2 \pi \nu_{i}(L-1) T / 2}\right]^{T}$ is the corresponding temporal signature vector.

\section{Sparse Estimation of Space-Time Clutter SPECTRUM}

After the direct-path component is removed, the overall output of the matched filter is the summation of three terms described in the previous section, and is expressed as

$$
\tilde{\mathbf{z}}^{(n)}=\tilde{\mathbf{z}}_{s}^{(n)}+\tilde{\mathbf{z}}_{c}^{(n)}+\tilde{\mathbf{z}}_{n}^{(n)} .
$$

Rewrite it as the product of a dense dictionary matrix $\tilde{\boldsymbol{\Phi}}$ and sparse vector $\tilde{\mathbf{u}}^{(n)}$, corrupted by additive noise vector $\tilde{\mathbf{z}}_{n}^{(n)}$, as

$$
\tilde{\mathbf{z}}^{(n)}=\tilde{\boldsymbol{\Phi}} \tilde{\mathbf{u}}^{(n)}+\tilde{\mathbf{z}}_{n}^{(n)}=\tilde{\boldsymbol{\Phi}}\left(\tilde{\mathbf{u}}_{s}^{(n)}+\tilde{\mathbf{u}}_{c}^{(n)}\right)+\tilde{\mathbf{z}}_{n}^{(n)},
$$

Our objective is to estimate the Doppler-angle characteristics of the clutter components $\tilde{\mathbf{u}}_{c}^{(n)}$ from the measurement vector $\tilde{\mathbf{z}}^{(n)}$. Note that the dimension of $\tilde{\mathbf{u}}^{(n)}$ is $K=N_{d} N_{s}$, where $N_{d}$ and $N_{s}$ are, respectively, the number of Doppler and azimuth grids defined in the Doppler-angle domain. 


\section{A. Complex Formulation Exploiting Persymmetry Property}

An existing approach to handle the underlying complex problem using the real-valued BCS is to cast it into a realvalued problem by separately considering the real and imaginary components [18]. In this case, (11) becomes

$$
\mathbf{z}^{(n)}=\mathbf{\Phi} \mathbf{u}^{(n)}+\mathbf{z}_{n}^{(n)},
$$

where $\mathbf{z}^{(n)}=\left[\operatorname{Re}\left(\tilde{\mathbf{z}}^{(n)}\right)^{T}, \operatorname{Im}\left(\tilde{\mathbf{z}}^{(n)}\right)^{T}\right]^{T}, \mathbf{z}_{n}^{(n)}=\left[\operatorname{Re}\left(\tilde{\mathbf{z}}_{n}^{(n)}\right)^{T}\right.$, $\left.\operatorname{Im}\left(\tilde{\mathbf{z}}_{n}^{(n)}\right)^{T}\right]^{T}, \mathbf{u}^{(n)}=\left[\operatorname{Re}\left(\tilde{\mathbf{u}}^{(n)}\right)^{T}, \operatorname{Im}\left(\tilde{\mathbf{u}}^{(n)}\right)^{T}\right]^{T}$, and $\boldsymbol{\Phi}=$ $\left[\begin{array}{lr}\operatorname{Re}(\tilde{\boldsymbol{\Phi}}) & -\operatorname{Im}(\tilde{\boldsymbol{\Phi}}) \\ \operatorname{Im}(\tilde{\boldsymbol{\Phi}}) & \operatorname{Re}(\tilde{\boldsymbol{\Phi}})\end{array}\right]$. As such, the $K \times 1$ unknown complex vector $\tilde{\mathbf{u}}^{(n)}$ is converted to a $2 K \times 1$ real vector $\mathbf{u}^{(n)}$. Such a simple extension does not utilize the fact that the real and imaginary components of $\tilde{\mathbf{u}}^{(n)}$ are merely the projection of complex coefficients into two orthogonal axes. A modified general approach is developed in [19], [20] to enforce the group sparsity of the real and imaginary components of the complex unknowns. Alternatively, for radar systems that exploit ULA and uniform azimuth sampling, we can also utilize the persymmetry [21] to convert the problem into a simple format of real-valued problem that maintains the group sparsity.

For persymmetric $\tilde{\mathbf{\Phi}}$, i.e., $\tilde{\boldsymbol{\Phi}}=\mathbf{J}_{N L} \tilde{\mathbf{\Phi}}^{*}, \boldsymbol{\Phi}_{\mathbf{T}}=\mathbf{T} \tilde{\boldsymbol{\Phi}}$ is real, where

$$
\mathbf{T}=\frac{1}{\sqrt{2}}\left[\begin{array}{cc}
\mathbf{I}_{N L / 2} & \mathbf{J}_{N L / 2} \\
j \mathbf{I}_{N L / 2} & -j \mathbf{J}_{N L / 2}
\end{array}\right]
$$

when $N L$ is even (the expression for odd $N L$ can be found in [21]). As such, we can reformulate (11) as

$$
\mathbf{Z}^{(n)}=\boldsymbol{\Phi}_{\mathbf{T}} \mathbf{U}^{(n)}+\mathbf{Z}_{n}^{(n)}
$$

where $\mathbf{Z}^{(n)}=\left[\operatorname{Re}\left(\mathbf{T} \tilde{\mathbf{z}}^{(n)}\right), \operatorname{Im}\left(\mathbf{T} \tilde{\mathbf{z}}^{(n)}\right)\right], \mathbf{Z}_{n}^{(n)}=\left[\operatorname{Re}\left(\mathbf{T} \tilde{\mathbf{z}}_{n}^{(n)}\right)\right.$, $\left.\operatorname{Im}\left(\mathbf{T} \tilde{\mathbf{z}}_{n}^{(n)}\right)\right]$, and $\mathbf{U}^{(n)}=\left[\operatorname{Re}\left(\mathbf{T} \tilde{\mathbf{u}}^{(n)}\right), \operatorname{Im}\left(\mathbf{T} \tilde{\mathbf{u}}^{(n)}\right)\right]$. Note that the unknown variables are arranged as a $K \times 2$ matrix where the two columns respectively represent the real and imaginary parts of the clutter entries. This problem can be solved using group-sparsity based techniques. In this paper, we use the multi-task BCS [16] for enhanced reliability. The group sparsity formulation (14) halves the number of sparse entries and thus makes the problem more feasible.

When multiple secondary samples are available, e.g., $n=$ $1, \ldots, D$, we can stack these observations as the following $K \times$ $2 D$ real-valued group sparsity problem

$$
\mathbf{Z}=\left[\mathbf{Z}^{(1)}, \ldots, \mathbf{Z}^{(D)}\right]=\mathbf{\Phi}_{\mathbf{T}} \mathbf{U}+\mathbf{Z}_{n},
$$

where $\mathbf{Z}_{n}=\left[\operatorname{Re}\left(\mathbf{T} \tilde{\mathbf{z}}_{n}\right), \operatorname{Im}\left(\mathbf{T} \tilde{\mathbf{z}}_{n}\right)\right]$ and $\mathbf{U}=[\operatorname{Re}(\mathbf{T} \tilde{\mathbf{u}})$, $\operatorname{Im}(\mathbf{T} \tilde{\mathbf{u}})]$. Note that the columns of $\mathbf{U}$ are still likely to share the group sparsity. This assumption is particularly valid when only a small number of secondary samples are used.

\section{B. Clutter Estimation Using Multi-Task BCS}

Equation (15) implies $2 D$ parallel equations

$$
\mathbf{z}_{i}=\mathbf{\Phi}_{\mathbf{T}} \mathbf{u}_{i}+\mathbf{z}_{n, i}, \quad i=1, \ldots, 2 D,
$$

where $\mathbf{z}_{i}, \mathbf{u}_{i}$, and $\mathbf{z}_{n, i}$ are the respective $i$ th columns of $\mathbf{Z}, \mathbf{U}_{i}$, and $\mathbf{Z}_{n}$. For the noise components that are i.i.d. zero-mean Gaussian variables with unknown precision $\alpha_{0}$ (precision is the reciprocal of the variance), the likelihood function for parameters $\mathbf{u}_{i}$ and $\alpha_{0}$, based on the observed data vector $\mathbf{z}_{i}$, may be expressed as

$$
\mathrm{p}\left(\mathbf{z}_{i} \mid \mathbf{u}_{i}, \alpha_{0}\right)=\mathcal{N}\left(\boldsymbol{\Phi}_{\mathbf{T}} \mathbf{u}_{i}, \alpha_{0}^{-1} \mathbf{I}_{N L}\right) .
$$

On the other hand, $\mathbf{u}_{i}$ is characterized by the Gaussian distribution $\mathrm{p}\left(\mathbf{u}_{i} \mid \boldsymbol{\alpha}\right)=\prod_{k=1}^{K} \mathcal{N}\left(u_{i, k} \mid 0, \alpha_{k}^{-1}\right)$, where $\boldsymbol{\alpha}=$ $\left[\alpha_{1}, \cdots, \alpha_{K}\right]^{T}$, and $u_{i, k}$ is the $k$ th element of vector $\mathbf{u}_{i}$. In the multi-task BCS, the group sparsity of the scatterer coefficients is represented by the common hyperparameters $\boldsymbol{\alpha}$ which are shared by all the $2 D$ tasks. The mean and variance of the estimated $\mathbf{u}_{i}$ are respectively given by [16]

$$
\boldsymbol{\mu}_{i}=\alpha_{0} \boldsymbol{\Sigma}_{i} \boldsymbol{\Phi}_{\mathbf{T}} \mathbf{z}_{i}, \quad \boldsymbol{\Sigma}_{i}=\left(\alpha_{0} \boldsymbol{\Phi}_{\mathbf{T}}^{T} \boldsymbol{\Phi}_{\mathbf{T}}+\mathbf{A}\right)^{-1},
$$

where $\mathbf{A}=\operatorname{diag}\left(\alpha_{1}, \ldots, \alpha_{K}\right)$.

We refer to the multi-task BCS that does not utilize the persymmetry as complex-BCS (C-BCS), whereas when the persymmetry is used, we refer to it as persymmetry-BCS ( $\mathrm{P}$ BCS).

\section{Space-Time Adaptive Processing}

Once the clutter angle-Doppler profile is estimated at range bin $n$ as a sparse collection of $K_{0}$ nonzero complex entries, $\eta^{(n)}\left(\nu_{k}, \phi_{k}\right), k=1, \ldots, K_{0}$, the received clutter component is expressed as

$$
\hat{\mathbf{z}}^{(n)}=\sum_{k=1}^{K_{0}} \eta^{(n)}\left(\nu_{k}, \phi_{k}\right) \mathbf{h}\left(\nu_{k}, \phi_{k}\right) .
$$

Considering the independence between the clutter components and noise, the covariance matrix, therefore, is estimated as

$$
\hat{\mathbf{R}}_{\mathbf{z}}=\sum_{k=1}^{K_{0}}\left|\eta^{(n)}\left(\nu_{k}, \phi_{k}\right)\right|^{2} \mathbf{h}\left(\nu_{k}, \phi_{k}\right) \mathbf{h}^{H}\left(\nu_{k}, \phi_{k}\right)+\hat{\alpha}_{0}^{-2} \mathbf{I}_{N L},
$$

where $\hat{\alpha}_{0}$ is estimated from the multi-task BCS as described above. Thus, the weight vector is given as

$$
\mathbf{w}_{\mathrm{CS}}=\frac{\left(\hat{\mathbf{R}}_{\mathbf{z}}\right)^{-1} \mathbf{h}_{0}}{\mathbf{h}_{0}^{H}\left(\hat{\mathbf{R}}_{\mathbf{z}}\right)^{-1} \mathbf{h}_{0}}
$$

where $\mathbf{h}_{0}$ is the spatio-temporal signature of the hypothetic target.

\section{Simulation Results}

Consider a bistatic passive radar where the illuminator, located at $\mathbf{p}_{\mathrm{T}}=[10,20,0.2]^{T} \mathrm{~km}$, emits DVB-T signals with a carrier frequency of $850 \mathrm{MHz}$ and a bandwidth of $7.6 \mathrm{MHz}$. The receiver is equipped with an 10-element ULA with an inter-element spacing of half-wavelength. The initial position of the receiver is $\mathbf{p}_{\mathrm{R}}(0)=[0,0,6]^{T} \mathrm{~km}$, and its velocity is $v_{\mathrm{R}}=[100,0,0] \mathrm{m} / \mathrm{s}$. The matched filtering output yields 600 $\mathrm{Hz}$ of azimuthal sampling rate, and 32 azimuthal samples are used for processing. To study the performance of the proposed approach versus target velocity, we let the initial target position to be $\mathbf{p}_{\mathrm{P}}=[-5,10,0]^{T} \mathrm{~km}$.

In Fig. 3, we show the clutter profile in the angle-Doppler domain for the range gate under test. The angle-Doppler 


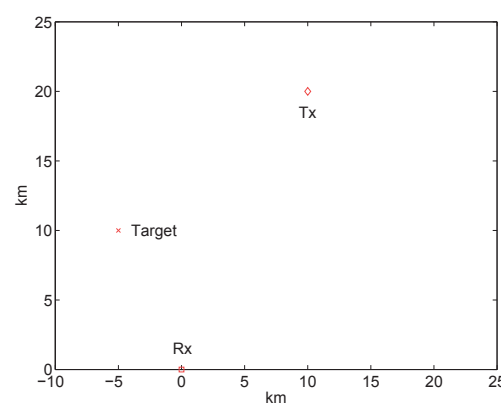

Fig. 2. Illustration of the scene.

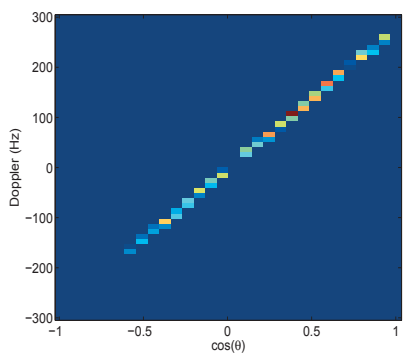

(a)

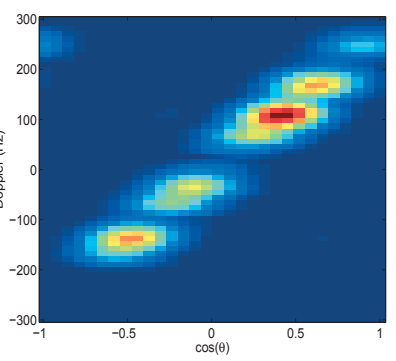

(b)
Fig. 3. Angle-Doppler domain clutter profile. (a) Estimated clutter profile; (b) Clutter profile obtained from Fourier-based method with a Hanning window.

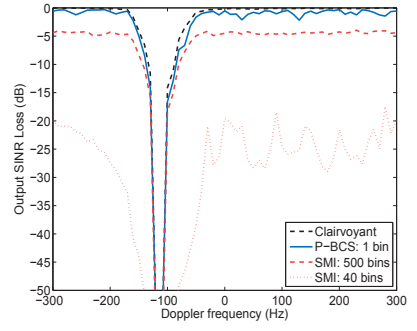

(a)

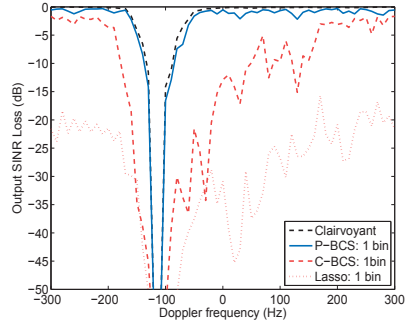

(b)
Fig. 4. Output SINR loss. (a) Comparison between Clairvoyant, P-BCS, and the SMI methods; (b) Comparison between P-BCS, C-BCS, and Lasso.

profile of the clutter is discretized into a grid of $N_{d}=60$ Doppler bins and $K_{s}=30$ angle bins. Fig. 3(a) depicts the estimated method, where the persymmetry-BCS is used. The input signal-to-noise ratio (SNR) is $-10 \mathrm{~dB}$, and the input clutter-to-noise ratio (CNR) is $40 \mathrm{~dB}$. For comparison, the Fourier-based estimation of the clutter profile is shown in Fig. 3 (b) with a poor resolution.

Figure 4(a) shows the output SINR loss compared to the optimal results computed in the no clutter case. The results are shown for the Clairvoyant, Persymmetry-BCS, and the SMI method with 500 and 40 secondary samples, respectively. With 500 secondary data samples, the SMI technique experiences a loss of approximately $3 \mathrm{~dB}$, which coincides with the RMB's rule. On the other hand, when the number of data samples is reduced to 40 , the performance loss becomes significant. The Persymmetry-BCS is obtained based on a single range bin data, and the performance is close to the Clairvoyant and better than the SMI with 500 secondary samples. In Fig. 4(b), we compare the performance of the persymmetry-BCS with complex-BCS and Lasso. It is evident that the latter two do not perform well because of inaccurate clutter profile estimations.

\section{CONCLUSiON}

We have examined the application of compressive sensing techniques for the implementation of space-time adaptive processing (STAP) in a bistatic passive radar. Our primary motivation is to enable effective clutter suppression in passive radar systems where a sufficient number of secondary data samples may not be available. Toward this end, we have chosen the Bayesian learning algorithm for effective clutter profile reconstruction and utilized the persymmetry property to align the real and imaginary components of non-zero clutter coefficients as group sparse entries.

\section{REFERENCES}

[1] N. J. Willis and H. D. Griffiths (eds.), Advances in Bistatic Radar, SciTech, 2007.

[2] R. Klemm, Principles of Space-Time Adaptive Processing. IEE, 2002.

[3] I. S. Reed, J. D. Mallett, and L. E. Brennan, "Rapid convergence rate in adaptive arrays," IEEE Trans. Aerosp. Electron. Syst., vol. 10, no. 6, pp. 853-863, Nov. 1974.

[4] M. C. Wicks, M. Rangaswamy, R. Adve, and T. B. Hale, "Space-time adaptive processing," IEEE Signal Proc. Mag., vol. 23, no. 1, pp. 51-65, 2006.

[5] X. Neyt, J. Raout, M. Kubica, V. Kubica, S. Roques, M. Acheroy, and J. G. Verly, "Feasibility of STAP for passive GSM-based radar," in Proc. IEEE Radar Conf., Veronna, NY, pp. 546-551, April 2006.

[6] J. Raout, X. Neyt, and P. Rischette, "Bistatic STAP using DVB-T illuminators of opportunity," in Proc. IET Int. Conf. Radar Systems, Edinburgh, UK, Oct. 2007.

[7] Y. D. Zhang and B. Himed, "Moving target parameter estimation and SFN ghost rejection in multistatic passive radar," in Proc. IEEE Radar Conf., Ottawa, Canada, April-May 2013.

[8] B. D. Carlson, "Covariance matrix estimation errors and diagonal loading in adaptive arrays," IEEE Trans. Aerosp. Electron. Syst., vol. 24, no. 4, pp. 397-401, July 1988.

[9] C. H. Gierull and B. Balaji, "Minimal sample support space-time adaptive processing with fast subspace techniques," IEE Proc. Radar, Sonar and Navig., vol. 149, no. 5, pp. 209-220, 2002.

[10] I. W. Selesnick, S. U. Pillai, K. Y. Li, and B. Himed, "Single-sample STAP using sparsity," in U.S./Australia Joint Workshop on Defense Applications of Signal Processing, Lihue, HI, 2009.

[11] K. Sun, H. Zhang, G. Li, H. Meng, and X. Wang, "Airborne radar STAP using sparse recovery of clutter spectrum," http://arxiv.org/pdf/1008.4185v1.

[12] Z. C. Yang, Z. Liu, X. Li, and L. Nie, "Performance analysis of STAP algorithms based on fast sparse recovery techniques," Progress In Electromagnetics Research B, vol. 41, pp. 251-268, 2012.

[13] B. Himed, Y. Zhang, and A. Hajjari, "STAP with angle-Doppler compensation for bistatic airborne radars," in Proc. IEEE Radar Conf., pp. 311-317, 2002.

[14] R. G. Baraniuk, "Compressive sampling," IEEE Signal Process. Mag., vol. 24, no. 4, pp. 118-124, July 2007.

[15] S. Ji, Y. Xue, and L. Carin, "Bayesian compressive sensing," IEEE Trans. Signal Proc., vol. 56, no. 6, pp. 2346-2356, June 2008.

[16] S. Ji, D. Dunson, and L. Carin, "Multitask compressive sensing," IEEE Trans. Signal Proc., vol. 57, no. 1, pp. 92-106, Jan. 2009.

[17] M. E. Tipping, "Sparse Bayesian learning and the relevance vector machine," J. Machine Learning Res., vol. 1, pp. 211-244, 2001.

[18] M. Carlin, P. Rocca, G. Oliveri, F. Viani, and A. Massa, "Directions-ofarrival estimation through Bayesian compressive sensing strategies," IEEE Trans. Antennas Propagat., vol. 61, no. 7, pp. 3828-3838, July 2013.

[19] Q. Wu, Y. D. Zhang, M. G. Amin, and B. Himed, "Complex multitask Bayesian compressive sensing," in Proc. IEEE ICASSP, Florence, Italy, May 2014.

[20] Q. Wu, Y. D. Zhang, M. G. Amin, and B. Himed, "Multi-static passive SAR imaging based on Bayesian compressive sensing, in Proc. SPIE Compressive Sensing Conf., Baltimore, MD, May 2014.

[21] G. Pailloux, P. Forster, J-P. Ovarlez, and F. Pascal, "Persymmetric adaptive radar detectors," IEEE Trans. Aerosp. Electron. Syst., vol. 47, no. 4, pp. 2376-2390, Oct. 2011. 